\section{Periphere arterielle Verschlusskrankheit: Sekundärprävention}

\section{H. Völler}

Klinik am See, Fachklinik für Innere Medizin, Abteilung für Kardiologie/Angiologie, Rüdersdorf

\section{Peripheral arterial disease (PAD): secondary prevention}

The prevalence of PAD rises as the population becomes increasingly older. At present, about 4.5 million citizens in Germany are estimated to be affected. In only one third of the patients intermittent claudication is found as a classical PAD marker. The course is thus predominantly asymptomatic. Consequently, careful evaluation of patients, including case history and pulse status as well as detailed recording of risk factors of atherosclerosis (chiefly smoking and diabetes mellitus), are important. When noninvasive methods of investigation is taken into account, the prevalence rises two- to three-fold. Duplex sonography with measurement of the ankle-brachial index $(A B I)$ is a method which has so far not been used widely although it is highly accurate. Life expectancy of patients with PAD is shortened by about ten years. The prognosis is determined by the natural course of the disease in only a few patients (ischemia syndrome and/or $\mathrm{ABI}<0.4$ ). Patients are at much greater risk for cardiovascular and cerebrovascular incidents than of a systemic disease. Mortality is raised up to six fold by concomitant coronary heart disease. Therapy should therefore not only be designed to treat the peripheral, as well as the atherosclerosis. Like walk training under supervision, no smoking, optimal glycemic control in diabetic patients and restoration of the lipid status to normal are treatment measures that have proved to be effective. As for pharmacotherapy, the efficacy of thrombocyte aggregation inhibitors is regarded as proved, whereas many drugs targeting symptoms have yielded disappointing results in patients with intermittent claudication.

Tab. 1 Beschwerdesymptomatik infolge pAVK und mögliche Differentialdiagnosen.

\begin{tabular}{|c|c|c|c|}
\hline & Symptomatik bei pAVK ${ }^{*}$ & \multicolumn{2}{|c|}{ Differentialdiagnose } \\
\hline & Typische Beschwerdesymptomatik & \multirow[t]{2}{*}{ Waden } & \multirow{2}{*}{$\begin{array}{l}\text { Venöse Insuffizienz } \\
\text { Chronisches Kompartment- } \\
\text { Syndrom } \\
\text { Nervenwurzelkompression }\end{array}$} \\
\hline Gruppe 1 & $\begin{array}{l}\text { Wadenschmerzen bei Anstrengung, } \\
\text { die nach 10-minütiger Ruhephase } \\
\text { nachlassen (Claudicatio intermittens) }\end{array}$ & & \\
\hline Gruppe 2 & $\begin{array}{l}\text { Beinschmerzen sowohl in Ruhe als } \\
\text { auch bei Belastung }\end{array}$ & $\begin{array}{l}\text { Hüftel } \\
\text { Schenkel }\end{array}$ & $\begin{array}{l}\text { Hüftgelenksarthrose } \\
\text { Spinalwurzelkompression }\end{array}$ \\
\hline & Atypische Beschwerdesymptomatik & \multirow[t]{3}{*}{ Füße } & \multirow{3}{*}{$\begin{array}{l}\text { Arthritis } \\
\text { Entzündliche Prozesse } \\
\text { (Buerger, Takayasu }\end{array}$} \\
\hline Gruppe 3 & $\begin{array}{l}\text { Beschwerden unter Belastung, } \\
\text { die nicht zum Abbruch zwingen }\end{array}$ & & \\
\hline Gruppe 4 & $\begin{array}{l}\text { Beschwerden, die weder in Ruhe } \\
\text { beginnen oder zum Abbruch der } \\
\text { Belastung führen, noch die Waden } \\
\text { betreffen oder nach } 10 \text {-minütiger } \\
\text { Ruhephase nachlassen }\end{array}$ & & \\
\hline
\end{tabular}

${ }^{*}$ Gruppenbildung in Anlehnung an den San-Diego-Fragebogen (Vasc Med 1996; 1: 65-71) und JAMA 2001; 286: 1599-1606
Als Ausdruck der systemischen Arteriosklerose ist der peripheren arteriellen Verschlusskrankheit (pAVK) eine größere Bedeutung beizumessen, zumal nicht so sehr die unteren Extremitäten als vielmehr das Leben der Patienten durch kardiovaskuläre Ereignisse gefährdet ist. In der hausärztlichen Versorgung ist jedoch nur jedem zweiten Arzt die Diagnose bewusst (JAMA 2001; 286:1317-1324). Während von Internisten in über $90 \%$ eine kardiale Anamnese erhoben wird, werden Fragen zur pAVK in weniger als $40 \%$ der Fälle gestellt (JVasc Circ 2000; 31:870-879). Einen niedrigen Stellenwert nimmt auch die Erhebung des Pulsstatus ein, wobei selten (ca. $10 \%$ ) eine weiterführende diagnostische Konsequenz gezogen wird.

Da der Großteil der Patienten mit pAVK asymptomatisch ist, liegen ungenaue epidemiologische Daten vor. Angaben zu Inzidenz und Prävalenz variieren in Abhängigkeit von der untersuchten Kohorte sowie dem eingesetzten diagnostischen Verfahren. Das klassische Beschwerdebild der Claudicatio intermittens als belastungsabhängige Muskelischämie weisen lediglich 10-30 \% der Patienten auf (JAMA 2001; 286:1317-1324; Arch Intern Med 1999; 159:387-392). Somit muss die Aufmerksamkeit auf die Erfassung anderer Funktionseinschränkungen der unteren Extremität gelenkt werden (Circulation 2000; 101:1007-1012). Diese belastungsabhängigen, definitionsgemäß nicht der Claudicatio intermittens zuzurechnenden Symptome sind bei jedem zweiten Patienten zu eruieren, aber differentialdiagnostisch von anderen Ursachen abzugrenzen (Tab. 1).

Eine hohe diagnostische Genauigkeit im Vergleich zur angiographisch gesicherten pAVK (Stenosen > $50 \%$ ) weist der Knöchel-Arm-Index (ABI) auf. Liegt der Quotient aus Blutdruckwerten, die oberhalb der Fußknöchel (Ankle) und an den Oberarmen (Brachial) gemessen werden unter 0,9 , beträgt die Sensitivität $>90 \%$, die Spezifität $\geq 95 \%$ (Tab. 2). 


\section{Epidemiologie}

Unter Berücksichtigung oben genannter Tatsachen erscheinen die in Bevölkerungsstudien erhobenen Zahlen zur Inzidenz mit 2/1000 pro Jahr für Personen unter 35 Jahre bis zu 7/1000 für solche über 65 Jahre das wahre Ausmaß der pAVK deutlich zu unterschätzen. Unter Verwendung von Befragungsbögen und klinischer Untersuchung liegt die Prävalenz altersabhängig zwischen $1 \%$ und $7 \%$, erfährt jedoch unter Einsatz der Dopplersonographie einen zwei- bis dreifachen Anstieg. Demzufolge weisen in der Rotterdam-Studie von 7715 Patienten älter als 55 Jahre 16,9 \% der Männer sowie 20,5 \% der Frauen (Arch Intern Med 2000; 160:2934-2938), im PARTNERS-Programm bis zu $29 \%$ der 6879 Patienten einen ABI-Index < 0,9 auf (JAMA 2001; 286:1317-1324).

\section{Prognose}

Diese Daten haben eine große prognostische Bedeutung, da auch asymptomatische Patienten ohne begleitende koronare Herzerkrankung im Zeitraum von 5 Jahren in 30 \% einen Myokardinfarkt, einen ischämischen Insult oder einen vaskulär bedingten Tod erleiden. Ist die pAVK klinisch manifest, so sterben im Verlauf der nächsten 10 Jahre je nach Studienpopulation bis zu 61,8 \% der Männer sowie 33,3\% der Frauen, wobei das Risiko 6,6fach erhöht ist, einen koronaren Tod $\mathrm{zu}$ erleiden ( $\mathrm{N}$ Engl J Med 1992; 326:381-386). Dagegen ist die Amputationsrate mit $2 \%$ bei Patienten mit Claudicatio intermittens im Verlauf von 5 Jahren gering. Bei kritischem Ischämiesyndrom erreicht sie allerdings in nur $3 \mathrm{Mo}$ naten 12,2 \% (Eur J Vasc Endo Vas Circ 1997; 14:91-95). Insgesamt sterben $55 \%$ der Patienten mit pAVK aufgrund der begleitenden koronaren Herzerkrankung, $10 \%$ aufgrund zerebrovaskulärer Ereignisse sowie $25 \%$ an anderen Ursachen. Weniger als $10 \%$ sterben an vaskulären Ereignissen, gewöhnlich im Rahmen eines rupturierten Aortenaneurysmas (J Vasc Circ 2000; 1 [Suppl]:1-296).
Tab. 2 Interpretation des Knöchel-ArmIndexes $(A B I) *$.

\begin{tabular}{|c|c|}
\hline$A B I$ & Ausmaß der pAVK \\
\hline$>1,30$ & Nicht komprimierbar** \\
\hline $0,91-1,30$ & Normal \\
\hline $0,41-0,90$ & $\begin{array}{l}\text { Leichte bis mäßige pAVK } \\
\text { (Claudicatio intermittens) }\end{array}$ \\
\hline $0,00-0,40$ & $\begin{array}{l}\text { Schwere pAVK (Ruheschmerz; } \\
\text { Gewebsuntergang) }\end{array}$ \\
\hline \multicolumn{2}{|c|}{$\begin{array}{l}\text { * der Knöchel-Arm-Index berechnet sich aus } \\
\text { Blutdruckwerten, die oberhalb der Fußknöchel } \\
\text { (Ankle) und an den Oberarmen (Brachial) } \\
\text { gemessen werden. } \\
{ }^{* *} \text { Ein Verhältnis > 1,30 weist auf verkalkte Gefäße } \\
\text { hin, so dass der wahre Druck nicht erhoben } \\
\text { werden kann. Bei falsch negativem Test ist eine } \\
\text { erweiterte Diagnostik, z.B. Puls-Volumen-Mes- } \\
\text { sung indiziert (N Engl J Med 2001; 344: } \\
\text { 1608-1621). }\end{array}$} \\
\hline
\end{tabular}

\section{Risikofaktoren}

Jedes zusätzliche Lebensjahrzehnt ist mit einem zwei- bis dreifach erhöhten Risiko verbunden, eine Claudicatio intermittens zu entwickeln. Gleiches galt für das männliche Geschlecht, wobei neuere Daten allerdings keine geschlechtsspezifischen Unterschiede mehr aufzeigen (Arch Intern Med 2000; 160:2934-2938, JAMA 2001; 286:1317-1324). Raucher tragen ein dreifach erhöhtes Risiko und sind im Vergleich zu Nichtrauchern darüber hinaus 10 Jahre früher von der Erkrankung betroffen. Dabei steigt die Schwere der Erkrankung mit der Anzahl gerauchter Zigaretten. Diabetes mellitus ist mit einem zweifachen Risiko behaftet, eine Makroangiopathie zu entwickeln. Eine optimale Blutzuckereinstellung kann nicht zweifelsfrei die Progression verhindern, was für die Nephro- und Retinopathie als Ausdruck der diabetischen Mikroangiopathie gesichert ist. Auch müssen Insulinresistenz, Glucoseintoleranz und Hyperinsulinismus als eigenständige Prädiktoren betrachtet werden. Dagegen besteht für die arterielle Hypertonie eine uneinheitliche Datenlage. Die kürzlich erschienene Rotterdam-Studie bestätigt jedoch den Bluthochdruck als unabhängigen Risikofaktor. In der Framingham-Studie war dieser bei Männern mit einem 2,5fachen, bei Frauen mit einem 3,9fachen Risiko verbunden, eine pAVK zu entwickeln. Die Dyslipoproteinämie ist ebenfalls ein bedeutender unabhängiger Risikofaktor, wobei die verschiedenen Parameter uneinheitlich bewertet werden. Einige Autoren schätzen das Gesamt-Cholesterin, andere das HDL-/Gesamt-Cholesterin-Verhältnis als unabhängigen Prädiktor ein. Die Hypertriglyzeridämie sowie auch das Lipoprotein(a) sind ebenfalls als eigenständige Risikofaktoren identifiziert worden. Neuerdings gibt es auch substanzielle Daten zur Bedeutung der Hyperhomocysteinämie, die bei der Entwicklung der pAVK im Vergleich zur koronaren Herzerkrankung ein 6,8fach vs 1 ,6fach erhöhtes Risiko aufweist. Zusammenfassend ist festzustellen, dass die Kombination von Risikofaktoren wie z. B. Rauchen, Diabetes mellitus und Bluthochdruck die Wahrscheinlichkeit verdreifacht, eine pAVK zu entwickeln.

Prävention

Im Rahmen der Primär- und Sekundärprävention sollten die eingesetzten Behandlungskonzepte vor allem den systemischen Charakter der Arteriosklerose berücksichtigen, um das Gesamtrisiko des Patienten zu reduzieren. Raucher sollten einen kompletten Nikotinverzicht üben und verhaltenstherapeutische sowie im Bedarfsfall auch pharmakologische Unterstützung erhalten. Häufig sind umfassendere Lebensstiländerungen geboten, die eine Kostumstellung sowie auch regelmäßige körperliche Aktivität beinhalten sollten. Hierdurch wird ein verbesserter Glucose-Metabolismus, eine Reduktion von LDL-Cholesterin und Triglyzeriden sowie eine höhere Rate der Nikotinabstinenz erzielt. Patienten mit 
den Symptomatik in der mit Simvastatin behandelten Gruppe im Vergleich zu Placebo um 38 \% reduziert werden konnte (Am J Cardiol 1998; 81:333-335). Da der Großteil von Patienten mit pAVK gleichzeitig an einer Koronar- oder Zerebralsklerose erkrankt ist, wird ein LDL-Cholesterinwert unter $100 \mathrm{mg} / \mathrm{dl}$ sowie ein Triglyzeridwert von weniger als $150 \mathrm{mg} / \mathrm{dl}$ empfohlen (JAMA 1999; 282:2051-2057). Obgleich die arterielle Hypertonie als unabhängiger Risikofaktor für die pAVK gilt, liegen keine gesicherten Daten über den Einfluss einer antihypertensiven Therapie auf die Progression der Erkrankung vor. Wichtig erscheint im Rahmen der generalisierten Arteriosklerose, dass mit dem Einsatz von ACE-Hemmern das Risiko ischämischer Ereignisse reduziert werden kann (N Engl J Med 2000; 342:145-153). Bei Patienten mit gleichzeitig vorliegender koronarer Herzerkrankung ist der Einsatz von $\beta$-Blockern gerechtfertigt, da keine Verschlechterung der Claudicatio intermittens zu erwarten ist. Selbstverständlich ist die Therapie mit Thrombozytenaggregationshemmer. Die niedrigdosierte Gabe von Acetylsalicylsäure ist in ihren Effekten belegt und bei fehlenden Kontraindikationen aufgrund der großen Preisdifferenz routinemäßig den ADP-Antagonisten, wie z. B. Clopidogrel vorzuziehen.

Wie bei der Diagnostik werden zurzeit auch die therapeutischen Ressourcen der pAVK nicht hinreichend genutzt. Im Vergleich zu Patienten mit KHK erhalten solche mit pAVK seltener Thrombozytenaggregationshemmer sowie eine antihypertensive oder cholesterinsenkende Therapie (JAMA 2001;286:13171324).

Fazit

Ein Großteil der Patienten mit pAVK wird aufgrund des asymptomatischen Verlaufs nicht diagnostiziert und somit im Hinblick auf ihr Gesamtrisiko unzureichend therapiert. Bei entsprechendem Risikoprofil ist neben detaillierter Anam- nese und Statuserhebung die Diagnose einer pAVK mit dem Knöchel-Arm-Index zu sichern. Die erforderlichen Therapiekonzepte sollten den systemischen Charakter der Arteriosklerose nicht länger ignorieren.

\section{Ansprechpartner}

Ansprechpartner für Patienten sind die Deutsche Gesellschaft für Angiologie, Gesellschaft für Gefäßmedizin e.V. (http://www.angiol.de und www.angio.de), die Deutsche Herzstiftung (info@herzstiftung.de) sowie die Deutsche Gesellschaft für Prävention und Rehabilitation von Herz- und Kreislauferkrankungen e.V., Friedrich-Ebert-Ring 38, 56068 Koblenz (http://www.dgpr.de).

Korrespondenz: Prof. Dr. H. Völler Klinik am See Fachklinik für Innere Medizin Abteilung für Kardiologie/Angiologie Seebad 84

15562 Rüdersdorf $b$. Berlin Tel.: 033638/78-623 Fax: 033638/78-624 heinz.voeller@klinikamsee.com

\section{Fachliche Betreuung der} „Serie Prävention“:

Prof. Dr. Dr. h. c. Peter C. Scriba Medizinische Klinik / Klinikum Innenstadt der Universität München

Ziemssenstr. 1

80336 München

Prof. Dr. Friedrich W. Schwartz Medizinische Hochschule Hannover Abteilung für Epidemiologie, Sozialmedizin und Gesundheitsforschung

Carl-Neuberg-Str. 1

30623 Hannover schlechterung einer bereits bestehen- 